lets approche de la phase d'application industrielle, pour ce qui regarde la stérilisation. Pour la mise en œuvre des autres radiations, de patientes recherches seront nécessaires, en vue de déterminer avec précision les possibilités d'emploi, et les résultats qu'il est légitime d'attendre de cette curieuse extension des méthodes de conservation des matières alimentaires.

\title{
BIBLIOGRAPHIE
}

[1] Voprossy Pitania, 1935, 4, 147.

[2] Journal Bact., 1942, 43, 125.

[3] Deutsche Molk, Ztg., 1941.

[4] Journal of Dairy Science, 1941, 24, 1055.

[5] Cold-ray pasteurisation of Milk. (Rapp. no 107 du British Intelligence Objectives Sub-Committee).

[6] Food Ind., 1947, 19, 1495.

[7] Milk Dealer, 1932, 21, 40.

[8] Journal Bact., 1946, 51, $\$ 87$.

[9] Deutsche Molk. Ztg., 1941, 45, 1052.

\section{ÉTUDE BACTÉRIOPHAGIQUE DU COLOSTRUM} par

\section{InèNe LIPSKA}

Au IVe Congrès de Microbiologie à Copenhague en 1947, j'ai eu la possibilité de rapporter les effets euratifs des bactériophages et j'ai indiqué les premiers essais préventifs contre les diarrhées d'été des nouveau-nés. Des bactériophages polyvalents furent administrés dans une première étude, le $4^{\mathrm{e}}$ et le $5^{\mathrm{e}}$ jour, et, dans une deuxième, le $2^{\mathrm{e}}$ et le $3^{\mathrm{e}}$ jour après la naissance. Une centaine de nouveau-nés, dans une clinique gynécologique ont reçu $2 \mathrm{~cm}^{3}$ des bactériophages polyvalents le $2^{\mathrm{e}}$ et le $3^{\mathrm{e}}$ jour. Après une année, ces nourrissons ont été visités par une même infirmière expérimentée pour constater leur état de santé. Les données furent que $71 \%$ n'avaient pas, pendant toute cette année, présenté de trouble digestif; $15 \%$ présentaient parfois des fèces moins moulés et $14 \%$ ont quitté Varsovie sans laisser d'adresse. Encouragée par ce bon résultat, j'ai recommencé à donner les bactériophages polyvalents, mais, cette fois, comme le premier liquide donné au nouveau-né. Dans une première période, j'ai ainsi traité soixante nouveau-nés ; encouragée, je crois devoir continuer ce travail.

Pour constater que le colostrum et le lait de la mère ne sont pas une source de bactériophages pour les nouveau-nés et n'ayant nulle part trouvé des études sur ce sujet, j'entrepris moi-même ce travail. 
Je vais faire un rapport sur le résultat des soixante analyses du premier colostrum des soixante mères et des soixante analyses des laits pris au moment où ces mères quittaient déjà la clinique, e'està-dire vers le dixième jour. L'étude bactériologique détaillée de ces soixante colostrums et de ces laits n'est pas encore finie. Les échantillons des colostrums et des laits pris aseptiquement toujours par la même infirmière furent tout de suite apportés au laboratoire où je les ensemençais pour l'étude bactériologique et pour l'examen bactériophagique. Tous les filtrats étaient préparés sur des bougies Chamberland 5 L 3. Pour éviter une infection par les bactériophages provenant de l'air du laboratoire où l'on opère tout le temps avec les virus des bactéries, j'ai eu le soin de faire toutes les filtrations dans des ballons stériles fermés au coton, pour les colostrums et pour les filtrats. Les filtrats vérifiés à l'étuve pour leur stérilité étaient étudiés sur la gélose et parallẹlement sur le bouillon dans une chambre stérile spécialement aménagée où chaque fois on pulvérisait de l'alcool car je n'ai pas eu une bonne stérilisation de l'air par les rayons ultra-violets.

Les filtrats furent étudiés avec les douze souches sensibles des colibacilles provenant des entérocolites aiguës des enfants et des eas chroniques des pyélonéphrites tous guéris par mes coliphages. La lecture des boîtes de gélose et des bouillons ensemencés avec des colibacilles et les filtrats des colostrums fut faite deux fois, après douze et vingt-quatre heures de séjour à l'étuve à $37^{\circ} \mathrm{C}$. Un second examen des filtrats fut fait avec les cinq souches pathogènes intestinales, à savoir : bact. dysent. Shiga, bact, paradys. Flexner, bact. typhiques, types $A$ et $E$ de Craigie et bact. paratyphique $B$, toujours en utilisant des boîtes de Petri et des bouillons pour avoir des résultats plus certains.

La troisième épreuve des filtrats des colostrums et des laits portait sur la recherche des bactériophages agissant sur les douze souches sensibles des staphylococcus aureus, citreus et albus sans pouvoir hémolysant du sang humain, isolés des cas des pyélonéphrites "et des furoncles guéris par les bactériophages. Toutes les souches sensibles des bactéries Gram positives et Gram négatives dans les conditions de mon étude ont donné des résultats négatifs. Dans les soixante colostrums examinés et les soixante laits pris des mêmes mères après une dizaine de jours, les bactériophages ne furent pas décélés.

Je veux indiquer les résultats d'étude bactériologique préliminaire de ces colostrums et de ces laits. Je me suis bornée à la constatation des bactéries Gram négatives et Gram positives.

Pour les soixante analyses des colostrums, j'ai trouvé : 
38 fois les bact. Gram -

33 fois les bact. Gram +

19 fois les bact. Gram + et Gram - à la fois

8 fois absence des bactéries.

Pour les soixante analyses des laits des mêmes mères j'ai constaté :

36 fois les bact. Gram -

39 fois les bact. Gram +

18 fois les bact. Gram - et Gram +

3 fois stérilité du lait.

Sur les boîtes de Pétri, pas une seule fois l'apparition da $B$. proteus ou des bactéries sporulés, c'est ce qui prouve que le prélèvement des échantillons fut bien fait.

\title{
REVUE
}

\section{LES CONGRESS DE L'AMERICAN CHEMICAL SOCIETY ET L'INDUSTRIE LAITIËRE}

\author{
par \\ G. GENIN \\ Ingénieur E. P. C.
}

Nous reproduisons ci-dessous l'analyse des différentes communications qui intéressent le lait et l'industrie laitière, présentées au cours des différents Congrès de l'American Chemical Society, organisés dans le courant des années 1949 et 1950 .

L'élimination des gaz contenus dans la crème glacée, par voie isothermique ou par voie isobarique: A. LACHMANN, E. L. JACK et D. H. Volman.

Un facteur important qui intervient dans la diminution du volume de la crème glacée et dans la contraction de ce produit qu'on observe au cours du magasinage ou de la distribution de ce produit, est la perméabilité de la crème et ses qualités mécaniques.

Différents échantillons de crème glacée, d'une composition détə̧rminée, ont été privés d'air, au cours de deux séries d'expériences, soit en abaissant la pression extérieure mais en opérant à température constante, à des températures comprises cntre -10 et $-50^{\circ}$, soit en élevant la température, mais en maintenant la pression fixe, à une valeur comprise entre 19 et $760 \mathrm{~mm}$. de mercure. Dans chaque 